\title{
Space-charge transport limits of ion beams in periodic quadrupole focusing channels
}

\author{
Steven M. Lund \\ Lawrence Livermore National Laboratory, Livermore, CA 94550, USA \\ Sugreev R. Chawla \\ Lawrence Berkeley National Laboratory, Berkeley, CA 94720, USA
}

\begin{abstract}
It has been empirically observed in both experiments and particle-in-cell simulations that space-charge-dominated beams suffer strong growth in statistical phasespace area (degraded quality) and particle losses in alternating gradient quadrupole transport channels when the undepressed phase advance $\sigma_{0}$ increases beyond about $85^{\circ}$ per lattice period. Although this criterion has been used extensively in practical designs of strong focusing intense beam transport lattices, the origin of the limit has not been understood. We propose a mechanism for the transport limit resulting from classes of halo particle resonances near the core of the beam that allow near-edge particles to rapidly increase in oscillation amplitude when the space-charge intensity and the flutter of the matched beam envelope are both sufficiently large. When coupled with a diffuse beam edge and/or perturbations internal to the beam core that can drive particles outside the edge, this mechanism can result in large and rapid halo-driven increases in the statistical phase-space area of the beam, lost particles, and degraded transport. A core-particle model is applied to parametrically analyze
\end{abstract}


this process. Extensive self-consistent particle in cell simulations are employed to better quantify space-charge limit and verify core-particle model predictions.

Key words: intense beam, space charge, emittance growth, simulation

PACS: 29.27.Bd, 41.75.-i, 52.59.Sa, 52.27.Jt

\section{Introduction}

The maximum transportable current density of an ion beam with high spacecharge intensity propagating in a periodic focusing lattice is a problem of practical importance[1,2]. Accelerator applications such as Heavy Ion Fusion (HIF), High Energy Density Physics (HEDP), and transmutation of nuclear waste demand a large flux of particles on target. A limit to the maximum current density can result from a variety of factors: instability of low-order moments of the beam describing the centroid and envelope, instability of higher order collective modes internal to the beam, growth in statistical phase-space area (rms emittance growth), excessive halo generation, and species contamination associated with issues such as the electron cloud problem. Simulations were first used to analyze the maximum current density transportable in quadrupole channels $[3,4]$ and provided guidance beyond initial heuristic estimates[5]. Experiments later obtained results consistent with simulations $[1,2]$.

The present work describes a promising new approach toward predicting the maximum transportable current density in a periodic quadrupole lattice due to intrinsic space-charge limits[6]. Previous studies to predict space-charge

Email address: SMLund@llnl.gov (Steven M. Lund). 
related transport limits in the absence of focusing errors and species contamination have not proved fully successful beyond a moment level description of low-order beam instabilities. Although moment-based centroid and envelope descriptions reliably predict regions of parametric instability where machines cannot operate[8,7], such models are overly optimistic when compared to simulations and experiments which observe degraded transport due to emittance growth and particle losses where the moment models predict stability $[1,2,3,4]$. On the other hand, higher-order collective mode theories based on the equilibrium KV distribution[10] predict broad parametric regions of instability where stability is observed in simulations with more realistic distributions[3,4] and in experiment[1,2]. The space-charge limit model proposed is based on particles oscillating outside, but near the beam edge exchanging energy with the oscillating space-charge field of a envelope matched beam core leading to increased particle oscillation amplitude, emittance blow up, and particle losses. This model can be applied to a wide range of matched core distributions and does not require an equilibrium core - which circumvents the practical problem of no smooth core equilibrium distribution being known. The increased understanding the origin of the observed limits obtained promises more reliable design of optimal intense beam transport channels.

We denote the phase advance of particles oscillating in a periodic focusing lattice in the presence and absence of beam space-charge by $\sigma$ and $\sigma_{0}$ (both measured in degrees per lattice period)[7,8]. The undepressed phase-advance $\sigma_{0}$ provides a measure of the strength of the linear applied focusing forces of the lattice that is relatively insensitive to the details of the lattice. $\sigma_{0}$ is generally made as large as beam stability will allow - because stronger focusing results in smaller beam cross-sectional area leading to smaller, more 
economical accelerator structures. $\sigma$ can be unambiguously defined by an rms equivalent, matched KV equilibrium beam[7,8] where all particles internal to the beam have the same phase advance. The ratio $\sigma / \sigma_{0}$ is a normalized measure of relative space-charge strength with $\sigma / \sigma_{0} \rightarrow 1$ corresponding to a warm beam with zero space-charge forces and $\sigma / \sigma_{0} \rightarrow 0$ corresponding to a cold beam with maximum space-charge forces. The maximum possible current density for a specified beam line-charge density will occur when $\sigma / \sigma_{0}$ is as small as possible.

Neglecting image charge effects, single particle and beam centroid oscillations are stable if $\sigma_{0}<180^{\circ}[8]$. The parameter space $\sigma_{0} \in\left(0,180^{\circ}\right)$ and $\sigma / \sigma_{0} \in$ $(0,1)$ can be regarded as potential machine operating points. Envelope models predict well understood bands of strong parametric instability when $\sigma_{0}>90^{\circ}$ and $\sigma / \sigma_{0}<1[7]$. The parameter region excluded by envelope instabilities for FODO quadrupole transport is indicated (in blue) on Fig. 1.

Considerations beyond centroid and envelope instabilities exclude further regions of $\sigma_{0}-\sigma$ parameter space. Transportable current limits based on preservation of beam statistical emittance and suppression of particle losses for a matched beam propagating in a periodic FODO lattice of 84 electric quadrupoles were experimentally studied by Tiefenback at LBNL[1,2]. It was found empirically that transport was stable (i.e., statistical emittance growth and particle losses below measurement thresholds) when

$$
\sigma_{0}^{2}-\sigma^{2}<\frac{1}{2}\left(120^{\circ}\right)^{2}
$$

The additional parameter region this criterion excludes for machine operation (partially overlapping the envelope band) is indicated (in red) on Fig. 1. For 
high space-charge intensity with $\sigma / \sigma_{0}<0.5$, this limit is more important than the envelope instability band because it is encountered first when approaching from low $\sigma_{0}$. The stability bound (1) has been applied by simply requiring that $\sigma_{0}<120^{\circ} / \sqrt{2} \simeq 85^{\circ}$. It is observed that transport becomes more sensitive to errors near the boundary of stability.

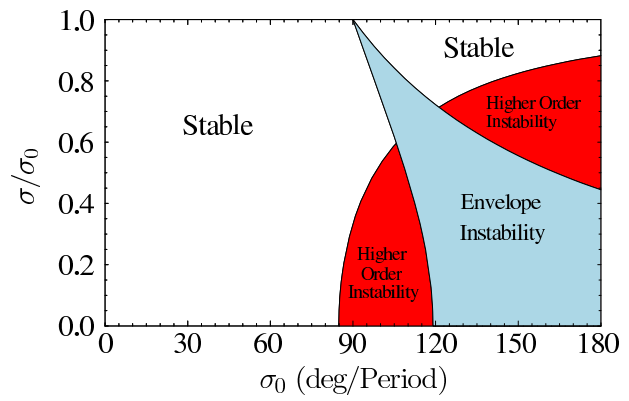

Fig. 1. (color online) Beam stability regions in a FODO quadrupole lattice. 


\section{Particle-in-Cell simulations}

Self-consistent electrostatic particle-in-cell (PIC) simulations have been carried out for a variety of initial beam distributions launched in a FODO quadrupole transport channel with $50 \%$ quadrupole occupancy $(\eta=1 / 2)$ and linear, piecewise-constant quadrupole forces. The transverse slice module of the WARP code[9] is employed to advance an initial transverse distribution with zero axial velocity spread. Applied focusing forces are adjusted for specified $\sigma_{0}$. Currents are adjusted for specified $\sigma / \sigma_{0}$ using fixed rms emittances $\left(\varepsilon_{x}=\varepsilon_{y}=50\right.$ mm-mrad). Numerical parameters are set for high resolution $(\geq 100$ radial zones across the beam core on a square mesh and $\geq 100$ residence corrected axial steps per lattice period) and good statistics ( $\geq 400$ particles per cell). A cylindrical beam pipe is large enough to suppress particle losses and image charge effects. Simulation results are in qualitative agreement with Eq. (1) for a wide variety of initial distribution functions. Initial distributions employed are rms matched transforms of continuous focusing equilibrium Waterbag and thermal distributions[8], KV, or semi-Gaussian distributions. This contrasts earlier work where Waterbag and Gaussian loads did not include space-charge screening effects and were far from initial force-balance[4]. Only the initial KV load employed is a true equilibrium of the periodic focusing channel. No exact, smooth equilibrium distributions are presently known for periodic focusing channels.

Parameters to the right of the stability bound [Eq. (1)] and to the left of the envelope instability band lead to statistical (rms) emittance growth and particle losses. $x$ - and $y$-plane average emittance $\left[\left(\varepsilon_{x}+\varepsilon_{y}\right) / 2\right]$ growth can be rapid and large as illustrated in Fig. 2(a) for a focusing channel with $\sigma_{0}=100^{\circ}$ 
and three initial distributions: semi-Gaussian, waterbag "equilibrium", and thermal "equilibrium." Much of this emittance growth can be traced to particles that evolve significantly outside the beam core as evident from Fig. 2(b) which shows the fraction of beam particles which evolve (at one or more points) more than 1.25 and 1.5 times the statistical beam edge radius (i.e., $\sqrt{x^{2} / r_{x}^{2}+y^{2} / r_{y}^{2}}>1.25,1.5$ with $r_{x}=2\left\langle x^{2}\right\rangle^{1 / 2}$ and $r_{x}=2\left\langle x^{2}\right\rangle^{1 / 2}$ calculated from the evolving distribution). The similarity of the results for the three very different non-equilibrium distributions shows that processes degrading the beam are relatively insensitive to the form of the initial distribution in deeply unstable parameter regions. Laboratory beams are born off a source (injector) and subsequently manipulated to match into a transport channel and are unlikely to be any equilibrium form. More detailed analysis of the simulation results show that initial beam distortions leading to the statistical emittance growth are primarily near the edge of the beam and subsequently act to strongly perturb the core. Core perturbations are observed in both the local density and temperature profiles. These perturbations typically lack elliptical symmetry and rapidly oscillate into the core with excursions larger near the beam edge. Beam envelope matches are not significantly degraded in the initial stages of instability.

A large number of PIC simulations were carried out to better quantify parametric regions of instability. Plane averaged emittance growth contours in $\sigma_{0}$ and $\sigma / \sigma_{0}$ are shown in Fig. 3 for an initial semi-Gaussian distribution. Irregular grid simulation points are indicated with dots. All simulations are advanced for six undepressed betatron periods, which is sufficient for saturation in strongly unstable regimes. Near the stability boundary, emittance growth slows and growth factors increase with longer propagation distance. Colors 


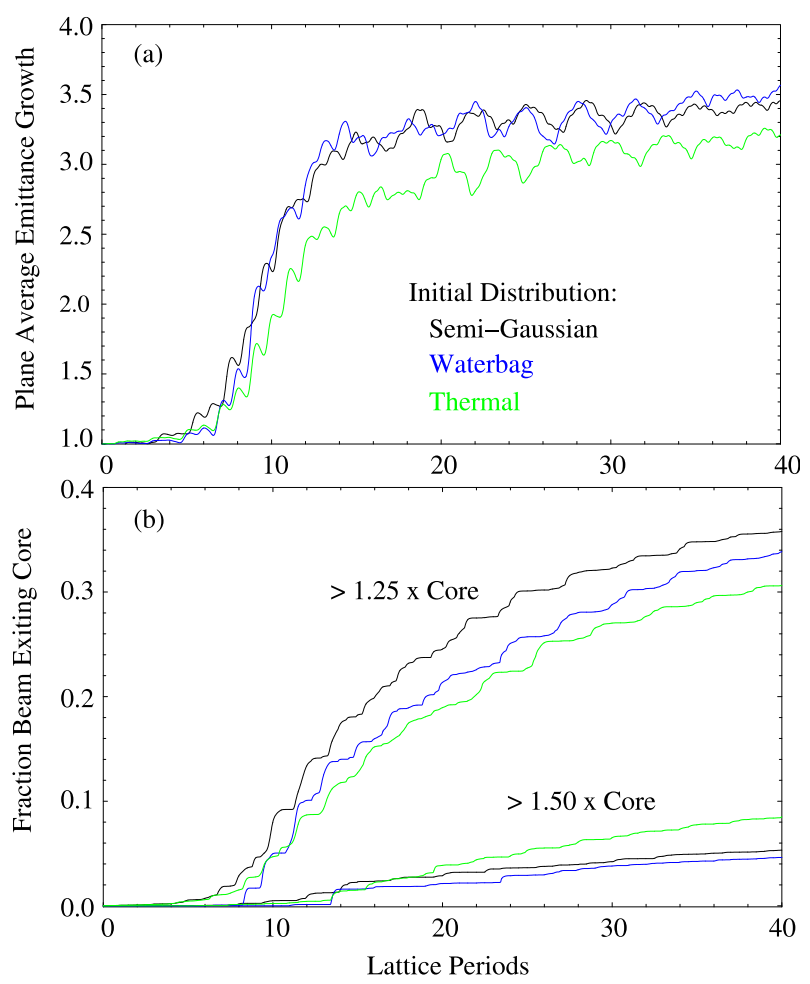

Fig. 2. (color online) PIC simulations of (a) the plane-averaged emittance growth for different initial distributions in a FODO quadrupole channel, and (b) the fraction of the beam distribution evolving outside the core. $\left(\sigma_{0}=100^{\circ}, \eta=0.5, L_{p}=0.5\right.$ $\mathrm{m}, \sigma / \sigma_{0}=0.2$, and $\left.\varepsilon=50 \mathrm{~mm}-\mathrm{mrad}\right)$.

show logarithmic scale emittance growth and $1 \%$ and $10 \%$ threshold contours (dashed) are labeled separately. The extent of the envelope instability band and Tiefenback's stability threshold are indicated. Results for initial waterbag and thermal distributions are similar, but the transition to instability has more structure for the waterbag distribution. Strong growth regions in all cases are qualitatively consistent with Tiefenback's threshold. Emittance growth cannot be attributed to KV-like modes internal to the beam[10,11]. Much of the emittance growth is associated with particles that evolve significantly outside the core (see Fig. 2b) rendering any linear internal mode interpretation questionable. Also, many KV modes are strongly unstable (instabilities exist for $\sigma / \sigma_{0}<0.3985$ even in the continuous focusing limit) where no rms emittance 
growth is observed. KV modes generally predict wrong parametric variations of instability (thresholds bend the wrong way). Large internal modes also possess little free energy to drive statistical emittance growth[8] and therefore may not be dangerous if they saturate at small amplitudes.

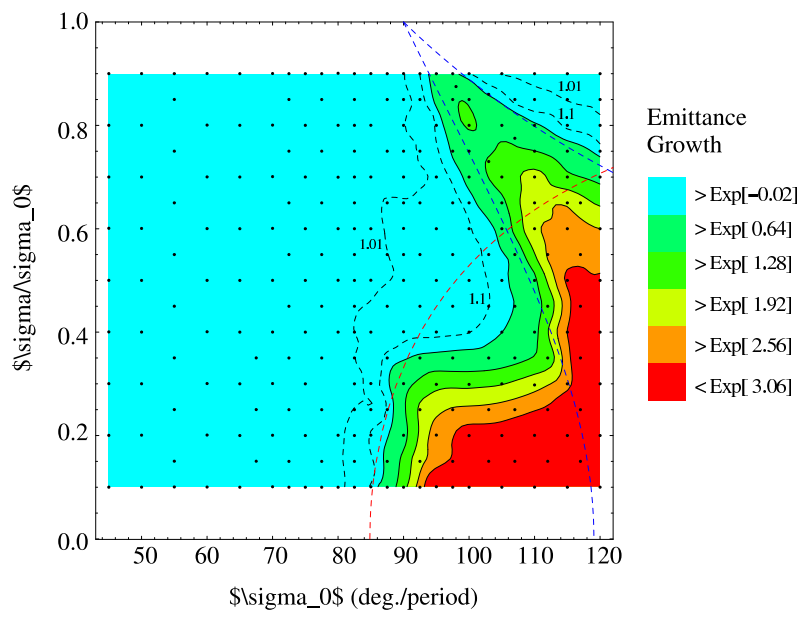

Fig. 3. (color online) Contours of emittance growth for an initial semi-Gaussian distribution in a FODO quadrupole channel. $\left(\eta=0.5, L_{p}=0.5 \mathrm{~m}\right.$, and $\varepsilon=50$ mm-mrad).

\section{Core-Particle model}

Consider an unbunched beam of ions of charge $q$ and mass $m$ propagating with axial velocity $\beta_{b} c$ ( $c$ is the speed of light in vacuuo) and relativistic factor $\gamma_{b}=1 / \sqrt{1-\beta_{b}^{2}}$. A linear applied focusing lattice is assumed, self-field interactions are electrostatic. Then the transverse orbit $x(s)$ of a beam particle evolves according to the paraxial equations of motion $[7,8]$

$$
x^{\prime \prime}+\kappa_{x} x=-\frac{q}{m \gamma_{b}^{3} \beta_{b}^{2} c^{2}} \frac{\partial \phi}{\partial x} .
$$

Here, $s$ is the axial coordinate of a beam slice, primes denote derivatives with respect to $s$, and $\kappa_{x}(s)$ is the linear applied focusing function of the lattice 
(specific forms can be found in Ref. [7]), and the electrostatic potential $\phi$ is related to the number density of beam particles $n$ by the Poisson equation $\nabla_{\perp}^{2} \phi=-q n / \epsilon_{0}$ in free-space. $\epsilon_{0}$ is the permittivity of free space.

The core of the beam is centered on-axis $(x=0=y)$, and is uniform density within an elliptical cross-section with edge radii $r_{j}$ (henceforth, $j$ ranges over both $x$ and $y$ ) that obey the KV envelope equations

$$
r_{j}^{\prime \prime}+\kappa_{j} r_{j}-\frac{2 Q}{r_{x}+r_{y}}-\frac{\varepsilon_{j}^{2}}{r_{j}^{3}}=0 .
$$

Here, $Q=q \lambda /\left(2 \pi \epsilon_{0} m \gamma_{b}^{3} \beta_{b}^{2} c^{2}\right)=$ const is the dimensionless perveance, $(\lambda=$ $q n(x=0, y=0) r_{x} r_{y}=$ const is the beam line-charge density), and $\varepsilon_{j}$ is the rms edge emittance along the $j$-plane. We take $\varepsilon_{j} \equiv \varepsilon=$ const. For a periodic focusing channel with lattice period $L_{p}, \kappa_{j}\left(s+L_{p}\right)=\kappa_{j}(s)$, the envelope is called matched when it has the periodicity of the lattice, i.e., $r_{j}\left(s+L_{p}\right)=$ $r_{j}(s)$. Undepressed particle phase advances are used to set the lattice focusing functions $\kappa_{j}$ using $\cos \sigma_{0}=(1 / 2) \operatorname{Tr} \mathbf{M}$ where $\mathbf{M}$ is the $x$ or $y$ plane transfer map of a single particle $(Q=0)$ through one lattice period. We take the $\kappa_{j}$ to be piecewise constant with occupancy $\eta \in(0,1]$. The matched beam envelope flutter varies only weakly with $\eta$ but increases strongly with increasing $\sigma_{0}$. The depressed particle phase advance is calculated as $\sigma=\varepsilon \int_{0}^{L_{p}} d s / r_{j}^{2}$.

It can be shown that the flutter of the matched beam envelope for periodic FODO quadrupole focusing systems with piecewise constant $\kappa_{j}(s)$ is given approximately (for $\sigma / \sigma_{0} \ll 1$ ) by $[12]$

$$
\frac{\left.r_{x}\right|_{\max }}{\bar{r}_{x}}-1 \simeq\left(1-\cos \sigma_{0}\right)^{1 / 2} \frac{(1-\eta / 2)}{2^{3 / 2}(1-2 \eta / 3)^{1 / 2}}
$$


Here, $\eta \in(0,1]$ is the occupancy of the quadrupoles in the lattice and $\overline{r_{x}}=$ $\left(1 / L_{p}\right) \int_{0}^{L_{p}} d s r_{x}$. Equation (4) shows that envelope flutter in a quadrupole channel depends strongly on $\sigma_{0}$ and weakly on $\eta$ (the variation in $\left.r_{x}\right|_{\max } / \bar{r}_{x}$ in $\eta$ is $\sim 13 \%$ ).

For a particle evolving both inside and outside the elliptical beam envelope, Eq. (2) can be expressed as

$$
x^{\prime \prime}+\kappa_{x} x=\frac{2 Q F_{x}}{\left(r_{x}+r_{y}\right) r_{x}} x
$$

with an analogous equation for the $y$-plane. Here, $F_{j}$ are form factors satisfying $F_{j}=1$ inside the beam $\left(x^{2} / r_{x}^{2}+y^{2} / r_{y}^{2} \leq 1\right)$ and $F_{x}=\left(r_{x}+r_{y}\right) \frac{r_{x}}{x} \operatorname{Re}[\underline{S}]$ and $F_{y}=-\left(r_{x}+r_{y}\right) \frac{r_{y}}{y} \operatorname{Im}[\underline{S}]$ outside the beam $\left(x^{2} / r_{x}^{2}+y^{2} / r_{y}^{2}>1\right) . \underline{S}$ is a complex variable defined as $\underline{S} \equiv \frac{\underline{z}}{\left(r_{x}^{2}-r_{y}^{2}\right)}\left[1-\sqrt{1-\frac{r_{x}^{2}-r_{y}^{2}}{\underline{z}^{2}}}\right]$, where $\underline{z}=x+i y$ and $i=\sqrt{-1}$. The particle equations of motion (5) are integrated numerically from initial conditions. We typically launch particles with initial $x$ and $y$ coordinates outside the beam edge (i.e., $x^{2} / r_{x}^{2}+y^{2} / r_{y}^{2}>1$ ) and with initial angles $x^{\prime}$ and $y^{\prime}$ consistent with coherent flutter motion of core particles extrapolated to the location of the particle, i.e., with $x^{\prime}=r_{x}^{\prime} x / r_{x}$ and $y^{\prime}=r_{y}^{\prime} y / r_{y}$. Diagnostics include particle trajectories, single particle emittances defined by $\epsilon_{x}=\sqrt{\left(x / r_{x}\right)^{2}+\left(x r_{x}^{\prime}-x^{\prime} r_{x}\right)^{2} / \varepsilon_{x}^{2}}\left(\epsilon_{x}=1\right.$ at the core distribution edge $)$, stroboscopic Poincare phase space plots, and particle oscillation wavelengths calculated from Fourier transforms of orbits. Particle trajectories and phasespaces analyzed in scaled units (e.g., with $x-x^{\prime}$ projections scaled as $x / r_{x^{-}}$ $\left.\left(x^{\prime} r_{x}-r_{x}^{\prime} x\right) / \varepsilon_{x}\right)$ to better illustrate oscillation extents relative to the matched beam core. 


\section{Core-Particle simulations}

To illustrate the halo structure, we launch particles along the $x$-axis of the elliptical beam in specified regions outside the beam edge (e.g., $x \in[1.1,1.2] r_{x}$ ) with zero incoherent angle spreads (e.g., $x^{\prime}=r_{x}^{\prime} x / r_{x}$ ). Fig. 4 illustrates $x-x^{\prime}$ Poincare phase-spaces for particles launched with $x \in[1.1,1.2] r_{x}$ for fixed $\sigma_{0}$ and two values of $\sigma / \sigma_{0}$ : (a) a high value (weak space-charge) well within the stable region of Fig. 1, and (b) a low value (strong space charge) in the unstable region. The Poincare strobe is one lattice period. Scaled coordinates $x / r_{x}$ and $\left(x^{\prime} r_{x}-x r_{x}^{\prime}\right) / \varepsilon_{x}$ are plotted to remove envelope flutter. The extent of the core is plotted in red. Extrapolations of the range of initial launch conditions are indicated in red based on the annular elliptical region formed if the initial particle conditions evolved with constant single-particle emittance $\epsilon_{x}$. Note the large change in scale between the stable and unstable plots. For the stable case, particles diving in and out of the matched envelope remain close to the initial launch range and indicate a weak, high-order resonance. For the unstable case, numerous resonances near the core become stronger and overlap causing the region immediately outside the core to break up into a stochastic sea that closely approaches the core. A large, 4-lobe bounding resonance (KAM surface) persists that ultimately limits the achievable particle oscillation amplitude. The phase advance of particles moving outside the envelope is strongly amplitude dependent ranging from $\sigma$ for amplitudes at the core boundary to $\sigma_{0}$ for very large amplitudes. Strong space charge $\left(\sigma / \sigma_{0} \ll 1\right)$ and large matched envelope oscillations (large $\sigma_{0}$ ) provide a strong pump at the lattice frequency. Numerous harmonics of particle orbits near the core resonate with the lattice resulting in overlapping resonances that produce a 
strongly chaotic region that approaches the core. This chaotic sea allows particles near the core to rapidly evolve to large amplitudes.
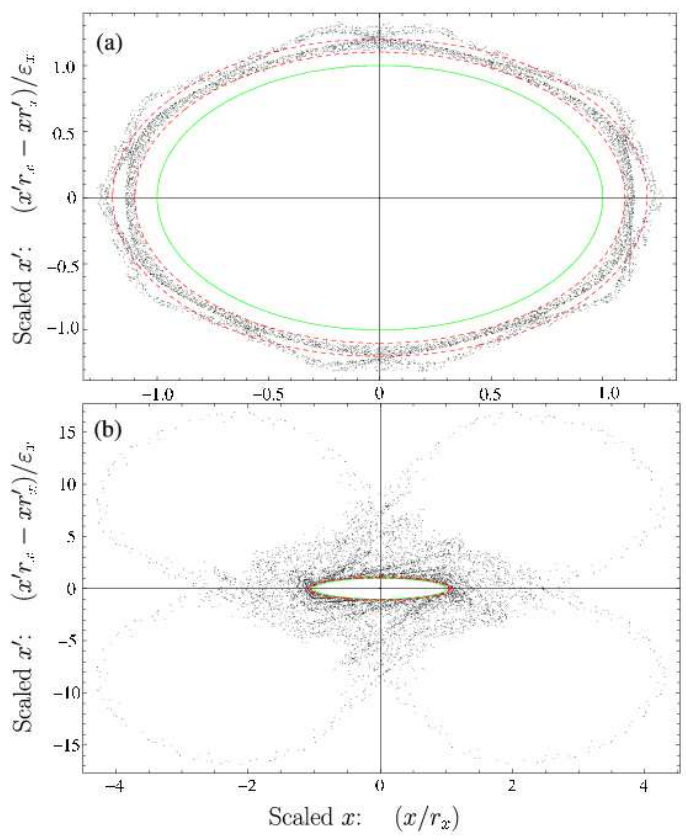

Fig. 4. (color online) Core-particle Poincare phase-spaces for $\sigma_{0}=100^{\circ}, \sigma / \sigma_{0}=0.67$ (a), and $\sigma / \sigma_{0}=0.2(\mathrm{~b})$. ( $\left.L_{p}=0.5 \mathrm{~m}, \eta=0.5, \varepsilon=50 \mathrm{~mm}-\mathrm{mrad}\right)$.

A new stability criterion is adopted to estimate where chaotic halo orbits near the beam core can degrade transport. When varying $\sigma_{0}$ and $\sigma / \sigma_{0}$, we define the stability boundary to be the first point when approached from stable regions (low $\sigma_{0}$ ) where particle groups launched near the core (e.g., $x \in[1.05,1.10] r_{x}$ ) experience large increases in oscillation amplitude (e.g., Max $\left[x / r_{x}\right]$ increased to 1.5). Boundary points obtained when particles launched with $x / r_{x} \in[1.05,1.10]$ increase in amplitude by factors of 1.5 (triangles) and 1.4 (squares) are plotted in Fig. 1. The boundary roughly tracks the region of strong emittance growth observed in experiment and simulations until the envelope instability band is approached. Results are relatively insensitive to the choice in initial group radius and amplitude increase factor. Earlier work by Langiel[13] employed a core-particle model to analyze transport limits but implied overly 
pessimistic stability criteria $\left(\sigma_{0}<60^{\circ}\right.$ and $\left.\sigma / \sigma_{0}>0.4\right)$ seemingly based on rough resonance overlap estimates.

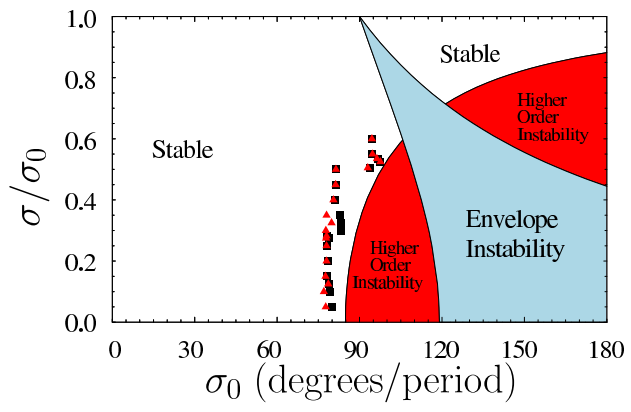

Fig. 5. (color online) Beam stability boundary calculated from a core-particle model for a FODO quadrupole channel. ( $\left.L_{p}=0.5 \mathrm{~m}, \eta=0.5, \varepsilon=50 \mathrm{~mm}-\mathrm{mrad}\right)$.

Halo properties analyzed persist when particles have finite angular momentum (not launched on-axis). Particles that leave the core in self-consistent PIC simulations generate similar Poincare plots to the core-particle model for a variety of initial distributions. Single particle emittance growths of $\sim 50$ are possible for particles near the beam edge that enter the halo in unstable 
regions. If a significant number of near-edge particles enter the halo, this can result in strong increases in rms beam emittance and distortions in the beam phase-space (both total and core). Particles leaving the core in unstable regions rapidly grow in amplitude over a relatively small number of lattice periods consistent with PIC simulations. Moreover, as observed in simulations and experiment, this halo induced mechanism for transport degradation is consistent with increasing sensitivity to the beam distribution and edge perturbations as the threshold region is approached. The core-particle model assumption of a uniform density elliptical beam core is reasonable for strong space-charge due to Debye screening and phase-mixing of initial perturbations. No periodic, nonuniform density equilibria are known and core perturbations are observed in PIC simulations to collectively evolve and disperse leaving smaller residual fluctuations and a rounded beam edge. Hence the uniform core model can provide a good approximation to the average impulse a halo particle experiences while traveling through the oscillating core. If the edge of the beam distribution is not sharp, as is expected for finite $\sigma / \sigma_{0}$, a significant population of edge particles can enter the halo and be elevated to large amplitudes in unstable regions. Due to envelope flutter, the spatial average temperature $\bar{T}_{x}$ of a beam with constant emittance $\varepsilon_{x}$ will vary as $\bar{T}_{x} \propto \varepsilon_{x}^{2} / r_{x}^{2}$. Thus, in a matched beam envelope the temperature will oscillate with the period of the lattice $\left(360^{\circ}\right.$ phase advance), decreasing where the envelope contracts and conversely decreasing where the envelope expands. For high $\sigma_{0}$ these fluctuations will tend to increase leaving the edge out of force-balance. On the other hand, the plasma response of the beam will have characteristic collective phase advance $\sigma_{p}=\frac{180^{\circ}}{\pi} \frac{L_{p}}{r_{x}} \sqrt{2 Q}$ This frequency will generally be incommensurate with and much slower than the temperature oscillations showing showing that the edge of the beam will have a more difficult time readapting to the focusing kicks 
as $\sigma_{0}$ increase and envelope flutter becomes larger.

\section{Conclusions}

A core-particle model was used to analyze the previously unexplained origin of space-charge related transport limits of beams propagating in periodic quadrupole focusing channels. It was shown that when matched beam envelope oscillations and space-charge strength are both sufficiently large, nearedge particles oscillating both inside and outside the matched beam envelope become chaotic and can experience large increases in oscillation amplitude. This resonance halo need not be tenuous and is distinct from envelope mismatch driven halo[14] because the driving oscillation is the fast flutter of the matched beam envelope rather than envelope mismatch modes. The matched envelope flutter becomes larger with increasing $\sigma_{0}$, providing a strong pump that further increases as beam space-charge forces become larger. Envelope oscillations also drive large temperature oscillations in the core of the matched envelope. Because the collective response of the beam to local force imbalances scales with the plasma frequency which is low relative to the lattice frequency, it is unlikely that the beam edge can consistently adapt. Lack of edge selfconsistency in periodically focused beam distributions makes it plausible that many near-edge particles can move sufficiently outside the beam core to partake in the resonance. Consequently, large distortions in the beam phase-space and large rms emittance growth can result. Stability thresholds based on this resonance picture are in rough agreement with experimental measurements and simulations. Analogous transport limits to the ones studied here will occur in other periodic focusing channels. Generally, unstable parameters will 
differ due to different scaling of matched beam envelope flutter. Envelope mismatch also increases driving envelope excursions and introduces additional frequencies - likely reducing the region of stable transport. Work is ongoing to further clarify the processes described. Further details of this work can be obtained on the arXiv e-print server[15] and in future publications.

\section{Acknowledgments}

B. Bukh and J. Barnard helped develop parts of the core-particle model. D. Grote aided the WARP simulations. J. Barnard, I. Haber, E. Lee, and P. Seidl provided useful discussions. This research was performed at LLNL and LBNL under US DOE contact Nos. W-7405-Eng-48 and DE-AC03-76SF0098.

\section{References}

[1] M.G. Tiefenback and D. Keffe, IEEE Trans. Nuc. Sci., NS-32, 2483 (1985).

[2] M.G. Tiefenback, Space-Charge Limits on the Transport of Ion Beams, U.C. Berkeley Ph. D thesis and Lawrence Berkeley Lab report LBL-22465 (1986).

[3] Simulations were carried out by Haber, Laslett, and colleagues that first suggested the limit. Partial results were reported in: I. Haber, IEEE Trans. Nucl. Sci. NS-26 3090 (1979); I. Haber and A.W. Maschke, Phys. Rev. Lett. 42, 1479 (1979).

[4] J. Struckmeier, J. Klabunde, and M. Reiser, Particle Accel. 15, 47 (1984).

[5] A.W. Maschke, Heavy Ion Space Charge Limits, Technical Report BNL 20297, Brookhaven National Laboratory, July 1975. 
[6] S.M. Lund and S.R. Chawla, Space-Charge Transport Limits in Periodic Channels, Proceedings of the 2005 Particle Accelerator Conference, Knoxville TN, paper FPAP034.

[7] S.M. Lund and B. Bukh, PRSTAB 7024801 (2004).

[8] M. Reiser, Theory and Design of Charged Particle Beams, (Wiley, 1994), and references therein.

[9] D.P. Grote, A. Friedman, I. Haber, W. Fawley, and J.-L. Vay, Nuc. Instr. Meth. A 415428 (1998).

[10] I. Hofmann, L.J. Laslett, L. Smith, and I. Haber, Particle Accel. 13, 145 (1983).

[11] S.M. Lund and R.C. Davidson, Phys. Plasmas 5, 3028 (1998).

[12] E. P. Lee, Phys. Plasmas 9, 4301 (2002).

[13] J.-M. Lagniel, Nuc. Instr. Meth. A, 345405 (1994).

[14] R.L. Gluckstern, Phys. Rev. Lett. 73, 1247 (1994).

[15] S.M. Lund and S.R. Chawla, to be posted on http://www.arxiv.org, 